\title{
Multifunctional Whisker Arrays for Distance Detection, Terrain Mapping, and Object Feature Extraction
}

\author{
Aimee E. Schultz ${ }^{\mathrm{a}, \mathrm{b}}$, Joseph H. Solomon ${ }^{\mathrm{a}, \mathrm{b}}$, Michael A. Peshkin ${ }^{\mathrm{b}}$, Mitra J. Hartmann ${ }^{\mathrm{c}}$ \\ a. These authors contributed equally to the work and are listed alphabetically \\ b. Department of Mechanical Engineering; \\ c. Departments of Biomedical Engineering and Mechanical Engineering \\ Northwestern University, Evanston, Illinois 60208, USA \\ \{aes, joe-solomon, peshkin,m-hartmann\}@northwestern.edu
}

\begin{abstract}
Several species of animals use whiskers to accurately navigate and explore objects in the absence of vision. We have developed inexpensive arrays of artificial whiskers based on strain-gage and Flex Sensor technologies that can be used either in passive ("dragging") mode, or in active ("whisking") mode. In the present work we explore the range of functions that whisker arrays can serve on a rover. We demonstrate that when mounted on a rover, whisker arrays can (1) Detect obstacles and determine obstacle distance (2) Map terrain features (3) Determine ground and surface texture (4) Provide an estimate of rover speed (5) Identify "slip" of the rover wheels, and (6) Perform 3dimensional extraction of object shape. We discuss the potential use of whisker arrays on planetary rovers and as an investigative tool for exploring the encoding of sensory information in the nervous system of animals.
\end{abstract}

Index Terms - vibrissa, whisker, tactile, rat, somatosensory

\section{INTRODUCTION}

Tactile sensing serves as a natural complement to vision because it can operate in the dark, underground, in fog, in the very near field of view, or when reflections and glare prevent accurate visual assessment of an object. Whiskers are a particularly efficient method of encoding tactile information, and behavioral experiments have demonstrated that both rats and pinnipeds (e.g., sea lions) can use their whiskers to navigate and extract object and environmental features without the use of vision [1-5].

Artificial whiskers have been used in several studies to obtain information about the environment. Most commonly, whiskers have been used as binary (contactdetection) sensors to avoid obstacles during navigation [e.g., 6-9]. Less frequently, whiskers have been used to perform wall following $[10,11]$, or to extract object features such as shape [12-14] and texture [15-18]. For example, [12-13] demonstrated that a single whisker could be swept over an object to extract its shape, while [14] has highlighted a geometrical approach to object feature extraction with a whisker array. [15-18] have investigated the effects of using different whisker materials (including real rat whiskers) to extract a variety of surface textures.

A recent set of studies has focused on determining the optimal arrangement and lengths of whiskers for use in a navigation task on a mobile rover [19-20]. These studies made the perhaps-surprising observation that for optimal obstacle avoidance, longer whiskers should be placed front and center on the rover, with whisker lengths decreasing along the sides and toward the rear. This arrangement is exactly opposite to that found in the real animal, in which shorter whiskers are located in front and longer whiskers in back $[1,5]$. The authors suggested that this difference might be attributed to the multi-functionality of whiskers in the real animal. Animals use their whiskers for many functions other than obstacle avoidance, including exploration and object identification; the natural arrangement might reflect a compromise between these different functions.

One aim of the present work was to develop a mechanical whisker array with the potential to perform the variety of functions that whiskers might subserve in real animals, including object distance detection and feature extraction as well as obstacle avoidance. We developed two prototypes of artificial whisker arrays based on strain gage and Flex Sensor technology. In the first prototype, strain gages were mounted at the base of copper wire whiskers to obtain measures of whisker deflection, force, amplitude, and velocity. These arrays were used in passive ("dragging") mode in the experiments described, and are superior for obtaining measurements of surface texture. The second prototype was built with whiskers made of spring steel wire. A $\sim 2.5 \mathrm{~cm}$ Flex Sensor was mounted at the base of each whisker to measure curvature. We mounted eight such whiskers on an array, allowing them to "whisk" back and fourth in unison, actuated by a single AC servo motor. In the present work, we chose to use the strain gage whiskers for passive sensing and flex sensor whiskers for active sensing, but both technologies can be used in either mode.

The current work was partially motivated by NASA's requirement for more agile and autonomous rover navigation on remote planetary surfaces. To this end, we have explored the multi-functionality of whisker arrays on a rover, and demonstrated that they can be used to (A) Detect obstacles and determine obstacle distance (B) Map terrain features as the rover goes over land with different contour profiles (C) Determine ground and surface texture (D) Provide an estimate of rover speed, useful for the dead-reckoning algorithms that the current Mars rovers use for navigation (E) Determine when the wheels of the rover are "slipping," for example when the rover is stuck in sand (F) Perform 3-dimensional extraction of object shape.

\footnotetext{
"This work was partially supported by a grant to MJH from NASA's CICT/ITSR Revolutionary Computing Algorithms project and the Jet Propulsion Laboratory, California Institute of Technology, and a graduate fellowship to JHS from the Dept. of Cognitive Science at Northwestern University.
} 


\section{METHODS}

\section{A. Distance detection}

Obstacle distance detection and feature extraction were based on a linearized solution of the equation describing beam bending for large angles (the 'elastica' problem), which relates the curvature $\kappa$ of a deformable beam to the moment at its base [12,13]:

$$
\kappa=\frac{\frac{d^{2} y}{d x^{2}}}{\left[1+\left(\frac{d y}{d x}\right)^{2}\right]^{\frac{3}{2}}}=\frac{M}{E I}
$$

where

$$
M=\left\{\begin{array}{cc}
F(a-x) & 0 \leq x \leq a \\
0 & a<x \leq L
\end{array}\right.
$$

In (1), $F$ is the force exerted at a distance, $a$, from the base of a cantilever beam; $y(x)$ is the bending of the beam at each location, $\mathrm{x} ; E$ is the elastic modulus; and $I$ is the moment of inertia. For a circular cross section, $I=1 / 4 \pi r^{4}$, where $r$ is the radius.

We solved (1) analytically for small angles to determine the contact point, $a$, between the whisker and object, similar to $[12,13]$. Note that the solutions to (1) will be different for a tapered beam, as would be the case for a real whisker [21].

\section{B. Whisker Sensors}

We used two different types of whisker sensors in the present work. In our first prototype we mounted each whisker in the center of a thin metal plate and used strain gage rosettes (Omega Engineering, Stamford, CT) to measure the 2-D strains on the plate. The gages were positioned at $0^{\circ}, 90^{\circ}$ and $225^{\circ}$ in the rosette, and the whiskers were $19 \mathrm{~cm}$ long, $0.3 \mathrm{~mm}$ diameter copper wire. These arrays were used passively in our experiments.

For our second whisker array, we used commerciallyavailable "Flex Sensors" (Spectra Symbol), to measure curvature at the base of the whisker ( $\kappa$ in (1)). In our experiments we were concerned only with curvature at the base of the whisker; therefore, we trimmed each $11.5 \mathrm{~cm}$ Flex Sensor down to $2.5 \mathrm{~cm}$, and then attached them to the base of an $11.5 \mathrm{~cm}$ long, $0.5 \mathrm{~mm}$ diameter spring steel wire. These sensors were used in active arrays (Fig. 1).

\section{Whisker Actuation}

A design requirement for the active array was that each whisker rotate about its base, similar to whiskers found on real animals. To actuate individual whiskers at their base under the control of a single motor, we mechanically coupled four cylinders through a five-bar linkage system, as shown in Fig. 1. One of the cylinders is actuated by the motor. Two whiskers (with Flex Sensors at the base, as described above) are attached at different heights to each cylinder. The height differences prevent collisions during whisking and provide greater resolution in the $\mathrm{z}$ (height) dimension.

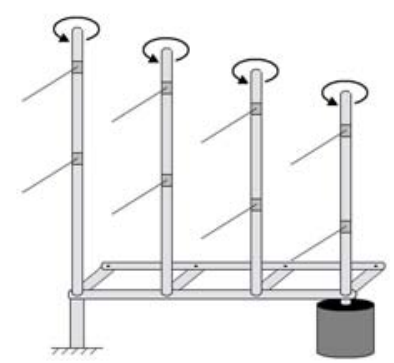

Fig. 1: Whisker Actuation. A 5-bar linkage system allows each whisker to be actuated at its base with only one motor.

\section{Whisker Array Calibration}

The Flex Sensors were calibrated at the beginning of each experiment by rotating the array and allowing each whisker to contact a peg placed at varying distances along its length. This allowed us to calibrate voltage to curvature and to appropriately zero the initial angle for each whisker.

The voltage signals from the strain gage whiskers were not calibrated on an absolute scale, as the present work required only their relative magnitudes and frequency responses. The strain gages were arranged in quarter bridge configurations. Each output was zeroed with a potentiometer before data acquisition. Any residual signal offset was zeroed in software.

\section{E. Rover Testbed and Data Analysis}

We mounted two "passive" whiskers (strain gage technology) on a Stampede ${ }^{\mathrm{TM}}$ remote-controlled car (Traxxas, Plano, Texas). For terrain mapping and texture extraction whiskers must come in contact with the ground, so they were mounted facing down. In addition, estimates of rover speed and wheel slip require the comparison of signals from a minimum of two whiskers traversing the same terrain some time apart. Thus, one whisker was mounted near the front of the rover and the other towards the back. While each whisker array was capable of holding up to eight whiskers, only one whisker was used on each array to obtain the preliminary results presented here.

The rover was geared down to move at speeds more typical of a Mars rover $(\sim 5 \mathrm{~cm} / \mathrm{sec})$. All data were acquired using a tether from the rover to a National Instruments BNC-2110 board connected to a Dell Inspiron 7500 laptop running Windows XP, and all data analysis was performed in MATLAB v.6.5 (2004; Mathworks, Nattick, MA).

\section{RESULTS}

A. Obstacle detection and object distance measurement

Equation (1) can be linearized and solved to show that object distance, $a$, is proportional to the ratio between rate of whisker rotation, $\dot{\theta}$, and the rate of curvature change at the whisker base upon or briefly after contact, $\dot{\kappa}$.

$$
a=C \frac{\dot{\theta}}{\dot{\kappa}}
$$

(Note that the same relationship also holds between torque and object distance [12]). Intuitively, nearby objects cause 
a greater rate of bending at the whisker base for a given rate of rotation, and our results confirm this.

Fig. 2 depicts the results of bringing a single whisker (Flex Sensor technology) into contact with a $3.2 \mathrm{~mm}$ diameter peg placed at different distances away from the whisker base. The figure clearly shows that the rate of change of curvature at the whisker base can be used to accurately extract object distance. When placed on a rover, these whiskers will thus not only provide a means to detect that an object is present, but also provide information about the distance to that object. In this way, a rover might not only avoid obstacles, but navigate closely around them.

\section{B. Mapping terrain features}

We used the distance sensing ability of the whisker arrays to map terrain features. Fig. 3(a) shows representative signals from a single whisker (strain gage technology) mounted near the front of the rover as it rolled over PVC tubes. Each trace in Fig. 3(a) illustrates a trial in which the rover rolled over four pieces of tube with equal diameter. The diameter was changed between trials. This experiment simulates a rover encountering terrain with different surface features. Inspection of Fig. 3(a) clearly shows that the larger diameter tubes caused a larger deflection of the whisker and hence a larger voltage response at the base of the whisker. Fig. 3(b) quantifies this result for 2 whiskers, demonstrating that the average amplitude of the peaks scales in a near-linear fashion with

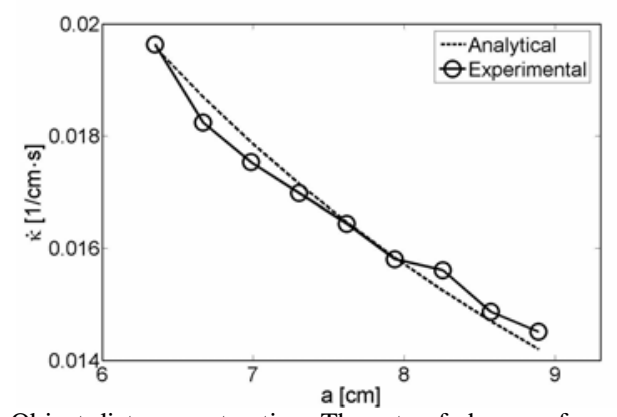

Fig. 2: Object distance extraction. The rate of change of curvature at the base of the whisker depends nearly linearly on object distance. (a)

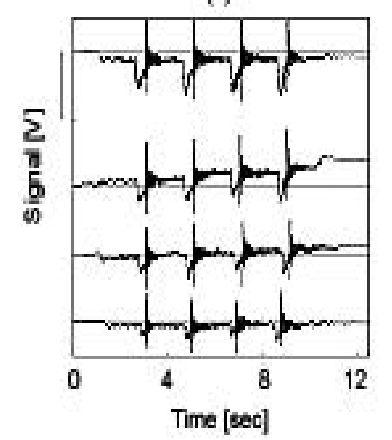

(b)

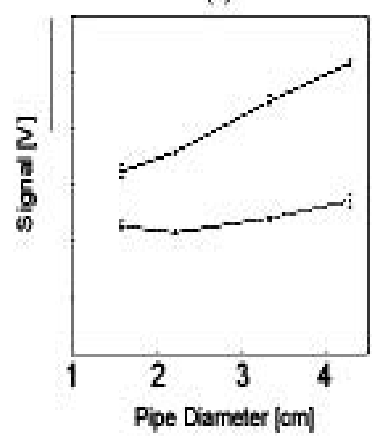

Fig. 3: Terrain mapping with whiskers. (a) Voltage at the base of a single whisker as the rover rolled over PVC tubes. Each for the four traces illustrates a trial in which the rover rolled over four pieces of tube with equal diameter, spaced approximately $60 \mathrm{~cm}$ apart. The diameter was changed between trials. From top to bottom, tube diameter, $\mathrm{d}=4.3,3.3$, 2.2 and $1.6 \mathrm{~cm}$. Scale bar $=1 \mathrm{~V}$. (b) Two whiskers independently measure tube height. Scale bar: $0.5 \mathrm{~V}$ top; $0.1 \mathrm{~V}$ bottom. tube diameter. Notice that the signal from one of the whiskers is much smaller than the other. This is because we placed different backing materials on each of the two strain gage rosettes for the purpose of investigating gain control (results not shown in the present paper).

Fig. 4 demonstrates that whiskers can extract terrain height as the rover rolls over "unknown" terrain. For this experiment we arranged the tubes in the following pattern: $\mathrm{D}, \mathrm{B}, \mathrm{C}, \mathrm{A}, \mathrm{B}, \mathrm{D}, \mathrm{B}, \mathrm{A}, \mathrm{C}, \mathrm{C}, \mathrm{D}, \mathrm{A}, \mathrm{D}, \mathrm{B}, \mathrm{C}, \mathrm{A}$, where $\mathrm{A}=1.6$, $B=2.2, C=3.3$, and $D=4.3 \mathrm{~cm}$. Figure 4 (a) shows a sample strain gage output resulting from a whisker being dragged over this configuration of tubes.

After letting the rover traverse the terrain, we used the whisker signals to determine the relative heights of the terrain features. In Fig. 4(b) we used the voltages from single whiskers (at the front and back of the rover) to estimate terrain height. Because the sensors are noisy, neither whisker alone provides unambiguous information about object height. In contrast, Fig. 4(c) shows the results of combining the signals from front and back whiskers to generate a more accurate terrain map. This example begins to illustrate the power implicit in using a parallel distributed sensor array: accuracy can be greatly increased by averaging over a larger number of sensors.

\section{Determining ground and surface texture}

We extracted surface texture information by examining the power spectrum of the signals as the whiskers encountered different surfaces. Texture information may be important when determining ground surface type or when determining the characteristics of a rock. Fig. 5 shows the running power spectrum from a single whisker (raw data overlaid in white) mounted near the front of the rover as it rolled over smooth laboratory flooring and grids of two different textures (grid $1=5.8$ ridges $/ \mathrm{cm}$; grid $2=0.6$ ridges $/ \mathrm{cm}$ ). The running power spectrum shown in Fig. 5 clearly distinguishes between the surfaces, similar to [17].
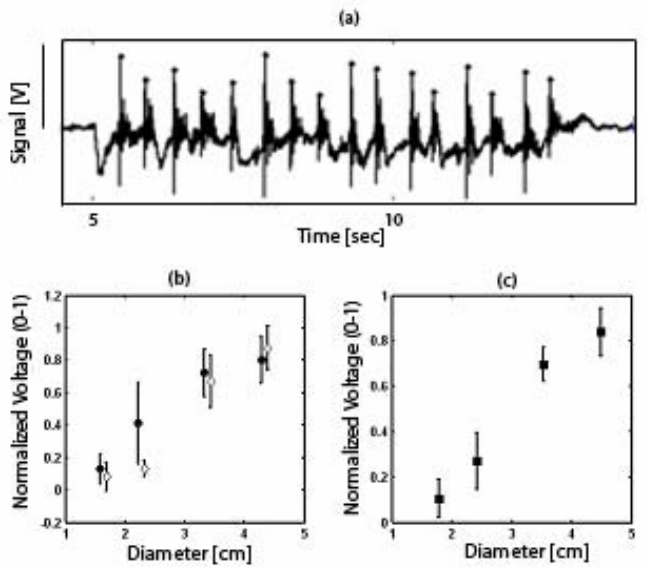

Fig. 4: Mapping unknown terrain with whiskers. (a) Sample raw data output of strain gage measuring the deflection of a single whisker traversing the terrain map. Scale bar $=1 \mathrm{~V}$. (b) Signals from a single whisker mounted near the front (diamonds) and back (circles) of the rover are insufficient to provide accurate height information. (b) Combining the signals from two whiskers greatly increases accuracy and provides nearly unambiguous information about object height. 


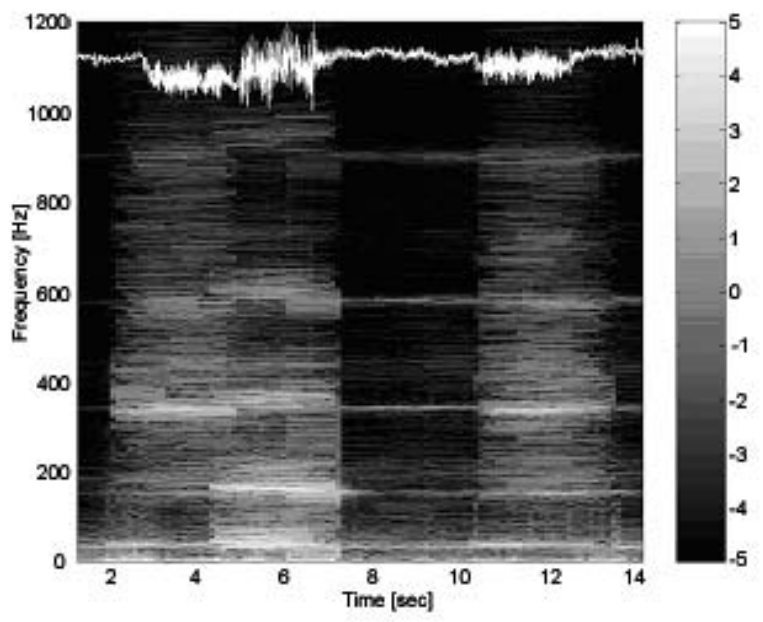

Fig. 5: Texture extraction with whiskers. Normalized raw voltage trace (white signal overlaid) and running power spectrum of the signal from the whisker as it goes over the following pattern of textures: smooth, grid 1, grid 2, smooth, grid 1, smooth. Colorbar indicates normalized spectral power $\left(\mathrm{V}^{2} / \mathrm{Hz}\right)$. Notice the presence of resonance frequencies even when traversing smooth ground, but the increase in amplitude when textures are encountered. In addition, the spectral characteristics are different between grid 1 and grid 2 .

\section{Estimation of rover speed}

We next used the cross-covariance between two whiskers mounted near the front and back of the rover to determine rover speed. Fig 6(a) shows two raw voltage traces from the two whiskers as a function of time. Again notice that the absolute amplitude of the voltage output from one of the whiskers is much smaller than the other; also note the presence of high frequency noise on the

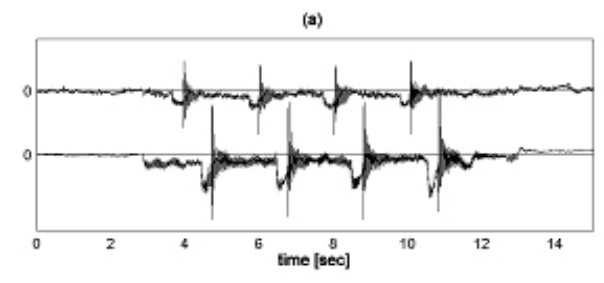

(b)

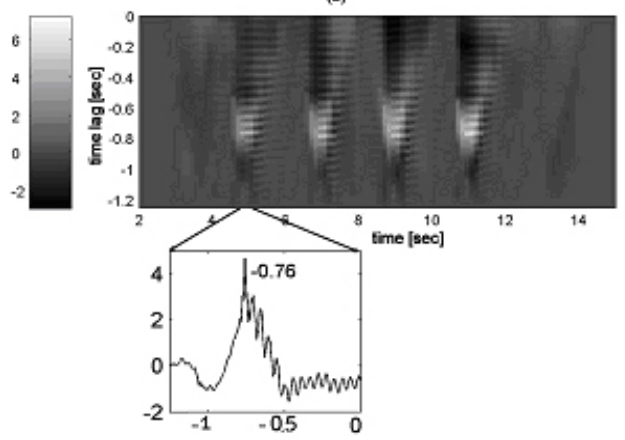

Fig. 6: Estimation of rover speed using whiskers. (a) Raw voltage signal from two exemplary front and back whiskers. Note consistent time delay between peaks as the rover traversed the terrain. Scale bar: top, $0.5 \mathrm{~V}$; bottom, $1 \mathrm{~V}$. (b) Performing a running cross-covariance reveals the temporal structure of the signals and an estimate of rover speed. Colorbar indicates magnitude of normalized cross-covariance. Expanded figure shows cross covariance at $\mathrm{t}=5 \mathrm{sec}$, illustrating the peak at a delay of -0.76 $\mathrm{sec}$. This corresponds to a rover speed of approximately $0.31 \mathrm{~m} / \mathrm{s}$, consistent with the actual speed. signal (likely due to vibrations partially associated with surface texture). Despite these highly non-ideal signal characteristics, Fig. 6(b) shows that a simple crosscovariance of the raw data is sufficient to extract an estimate of rover speed. More specifically, Fig. 6(b) shows that the running cross-covariance between the two raw data signals $(2.5 \mathrm{sec}$. windows, 1 second overlap) cleanly extracts the difference between the times of object contact of the two whiskers. Note that by performing a cross correlation between signals from whiskers separated laterally (side-to-side), we could also do terrain extraction in that dimension, in addition to front-to-back (data not shown).

The estimate of rover speed found using the crosscovariance of the signals was verified by comparing it to the actual speed of the rover, as determined by dividing the known distance between tubes by the average time between spikes in the signal. This result is shown in Fig. 7.

\section{E. Determine times of wheel slip}

Fig. 8 demonstrates that we can use our whiskers to determine the times when the rover wheels slip. Specifically, the top portion of Fig. 8 shows raw voltage traces from the front and back whiskers as a function of time. For the first part of the graph, the rover runs smoothly. At $\mathrm{t}=\sim 6$ seconds, the rover gets stuck and the wheels start to slip; the wheels are turning but the rover is not going forward. The signal from the whiskers changes abruptly to encompass a highly correlated low-frequency signal (with $\sim 0$ time delay between the whiskers), almost certainly corresponding to the frequency of wheel rotation.

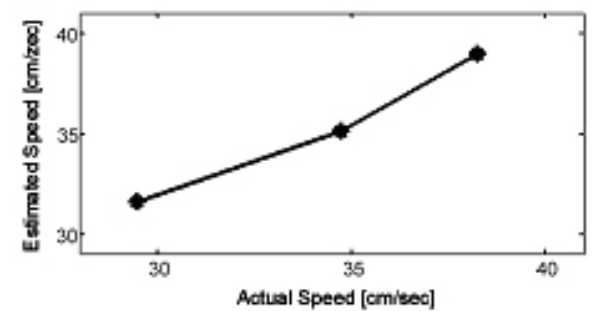

Fig. 7: Rover speed estimated from the signal cross-covariance provides an accurate estimate of actual rover speed.

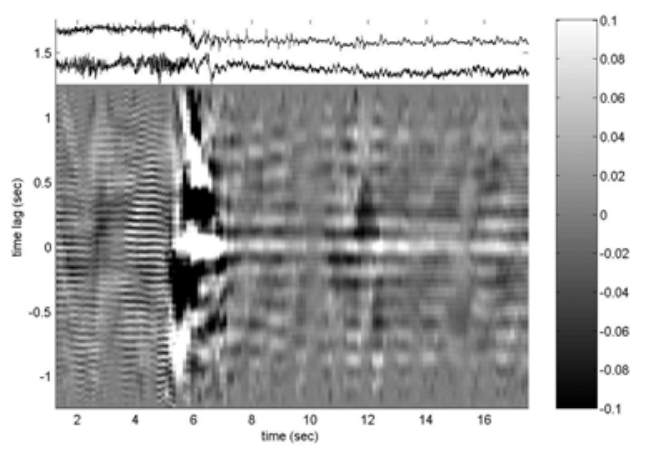

Fig. 8: Normalized raw voltage traces (black traces at the top) of the two whiskers as the rover's wheels start to slip. The running cross-covariance between the whiskers clearly shows a strong correlation (with zero time delay) between the two signals. Even without extensive data processing, the temporal structure is distinct from terrain features shown in Fig. 6. Colorbar indicates magnitude of normalized cross covariance. 
F. Perform object feature extraction, including the extraction of shape parameters

The results in part $A$ demonstrated that the point of whisker-object contact can be accurately determined based on measuring either torque or curvature at the base of a single whisker. It is now only a small step to use multiple whiskers to gather multiple contact points to extract object curvature information. Fig. 9 shows how whiskers can be used to extract the curvature from two cylinders of different diameters. Five "whisks" were performed per cylinder and the median taken in order to maximize accuracy.

In Fig. 9(a), the whiskers hit at their endpoints, while in Fig. 9(b) the whiskers hit along their length. Although the former case causes (2) to overestimate distance $a$, we can account for this by simply enforcing that $a$ must be less than or equal to the length of the whisker.

The cylinders have uniform curvature and no concave surfaces, making them much easier to analyse than most real-world objects. We demonstrated more complex feature extraction using a mannequin head. We fixed the center of the mannequin head relative to the center of the whisker array and then gathered whisker contact points at different head orientations ranging from $0^{\circ}$ (directly facing the array) to $70^{\circ}$, in increments of $5^{\circ}$. Note that only the area from the nose to the brow-ridge has been captured due to the size of the array. Fig. 10 shows that we are able to extract complicated contour information, including concavities. A smoothing spline was used to interpolate between contact points.

\section{DISCUSSION AND FUTURE WORK}

The present work demonstrates an important proof-ofconcept: whisker arrays mounted on a rover can perform multiple functions, including terrain mapping, texture and shape extraction, and characterization of rover movement. This multifunctional capability makes the whisker arrays ideal complementary sensors to vision (cameras) and audition (microphones). Future work will involve ensuring that the arrays can extract information uniquely and reliably.
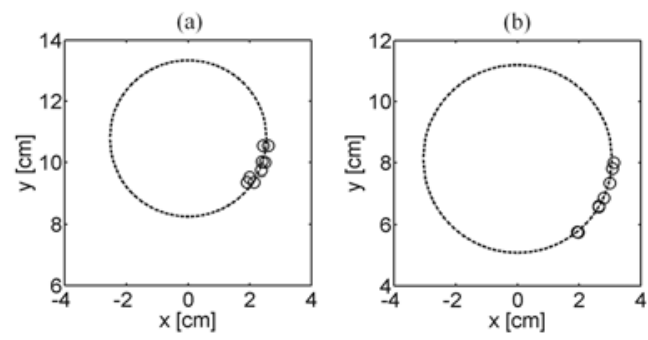

Fig. 9: Curvature extraction with whiskers. (a) Cylinder diameter $=7.6 \mathrm{~cm}$ (b) Cylinder diameter $=12.2 \mathrm{~cm}$.

(a)

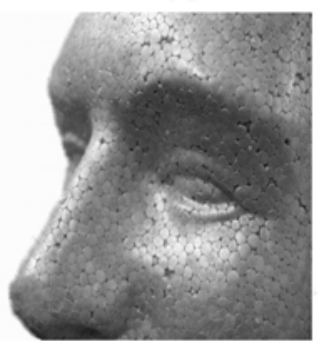

Fig. 10: (a) Actual mannequin head (b) Extracted shape. Black dots indicate contact points.
We anticipate, for example, that there may be "patterns of confusion" that result when the spatial frequencies of a particular terrain match the spectral characteristics of wheel slip. Figs 5, 6, and 8 suggest, however, that we can resolve this ambiguity by looking at cross-correlation and power spectra simultaneously. Our arrays may also provide an excellent testbed system for investigating neural coding, as we can now examine how the critical variables describing the state of the whisker array are most efficiently encoded. An important component of this work will involve examining the effects of different movements on the sensory signals acquired.

\section{A. Potential use of whiskers on autonomous rovers}

To efficiently explore an unknown environment, an autonomous rover such as those used in NASA missions must accurately determine its location within a landscape, avoid obstacles and hazards robustly and in real-time, and detect and explore objects of scientific interest. Whisker arrays could confer, robust, real-time hazard avoidance, even at high speeds, and improve maneuverability.

\section{B. Whiskers as a complementary sense to vision}

A whisker array functioning as a proximity-detector system can operate when vision is limited (in darkness, underground, or under an overhang), and/or when reflections, glare, or low-contrast prevent accurate visual assessment of an object. Additionally, unlike audition, whiskers do not need to emit a signal to be reflected off an object. Whiskers are mechanically flexible, and can be designed to be difficult to break. If a whisker should happen to break or kink, we have developed a method to sense such damage through analysis of resonant frequencies as well as a method to "heal" the whisker through extrusion of additional wire. Such ruggedness and reliability makes them ideally suited for the periphery of the rover, as well as for placement close to the ground. Whiskers can bend to permit the rover to get through narrow spaces. It is also known that whisker arrays can function effectively even if several whiskers are damaged [5], a design feature we aim to mimic with our artificial arrays.

As described in [22], range images generated by stereo vision cameras on remote rovers are generally not sufficient to determine a safe driving path. Field of view restrictions and error recovery behaviors can potentially force a rover to run into an unseen area $[22,23]$. To solve these problems, a "local map" is constructed of the terrain around the rover [22]. To ensure an appropriate safety margin for the rover, the appearance of an obstacle in the local map tends to grow beyond the obstacle's physical boundaries by half the vehicle width in all directions. This margin of safety means that rovers cannot access narrow passageways or crawl directly under boulders, and critical science data is clearly being lost. A whisker system used in real-time proximitydetection mode would complement data from cameras to ensure safe exploration while coming much closer to objects and landforms of scientific interest.

A whisker system could also help to ensure that navigation algorithms accurately identify the shortest path to an objective, even if the path is narrow. For example, a whisker system would allow the rover to drive through a pair of obstacles with less than 1.5 times the width of the rover between them, saving valuable time and resources. 
The whisker arrays could also enable a sequence in which the rover drives around the perimeter of an interesting rock (using whiskers for edge-following and contour tracing), taking images as it goes, in essence enabling a singlecommand-from-ground rock survey.

\section{Real-time navigation and object localization}

Current NASA rover navigation is a blend of wheel and visual odometry, sun-sensing, and inertial sensing [24]. Optical encoders on the wheels are used to determine how far the rover has traveled. However, because the wheels on the rover are subject to slip, this kind of encoding system is subject to cumulative errors of up to ten percent. As shown in Figs 6, 7, and 8, whiskers can provide an independent measure of rover speed and wheel slip. Even a simple binary whisker detection system, indicating contact with a landmark seen in previous imagery, could provide accurate updates for the dead-reckoning data.

\section{Terrain mapping and texture extraction}

Determining surface texture is a difficult problem for visual sensing systems and is often confounded by lighting conditions. A whisker system could extract texture information from object surfaces, and also ground texture (gravel, pebbles, smooth rock) as the rover traverses the landscape. The ability to determine mechanical soil properties before a rover rolls onto a surface would dramatically decrease risk to the rover. Texture information is particularly important to planetary geologists, who currently rely on visually-based texture information and elemental analysis to determine rock composition. However, the visual texture often does not bear a one-to-one relation to the rock's actual texture and grain size [25]. Artificial whiskers could accurately assess rock grain size by examining surface roughness, enabling rock identification and characteristics about the origins of the rock.

\section{E. Near-field imaging capability}

Near field imaging capability is likely to be particularly important to planetary geologists. The microscopic imager (MI) on board MER has a $3.1 \mathrm{~cm}^{2}$ field of view at a working distance of $6.3 \mathrm{~cm}$, and has $30 \mathrm{microns} /$ pixel resolution [26]. However, a serious limitation on the MI images is that they are $2 \mathrm{D}$, and cannot provide a topographic profile of the rock surface. Here, we have demonstrated that an array of artificial whiskers has the ability to discern complex object features. Whiskers could be used to accurately map the fine 3D spatial features of rocks, recognizing features that may be too small to visually detect. (e.g., a crack on a facet of the rock at an angle slanted relative to the MI imaging plane). Studies of analogous rocks on earth from the Mojave Desert have shown that these cracks often harbor microscopic lifeforms. If life is to be found on rocks on other planets, it is likely to be found in these types of microscopic cracks.

\section{ACKNOWLEDGMENTS}

The authors thank Matthew J. Wagner, an undergraduate at UIUC who geared down the Stampede and constructed the rover body. MJH thanks Christopher Assad, Brett Kennedy, and Robert Anderson at JPL for useful discussions.

\section{REFERENCES}

[1] S. B. Vincent, "The function of the vibrissae in the behavior of the white rat," Behavior Monographs vol 1, pp. 1-81, 1912.

[2] G. Carvell and D. Simons, "Task-related and subject-related differences in sensorimotor behavior during active touch," Somatosens. Mot. Res., 12:1-9, 1995

[3] G. Dehnhardt, B. Mauk, and H. Bleckmann, "Seal whiskers detect water movements," Nature 394:235:236, 1998.

[4] G. Dehnhardt, and G. Duker, "Tactual discrimination of size and shape by a California sea lion (Zalophus californianus)," Animal Learning and Behavior, vol. 24, pp. 366-374, 1996.

[5] M. Brecht, B. Preilowski, M. Merzenich, "Functional architecture of the mystacial vibrissae," Behav. Brain Res., vol. 84, pp. 81-97, 1997.

[6] R. A. Brooks, "A robot that walks; emergent behaviors from a carefully evolved network.," Neural Computation, vol. 1, pp. 253-262, 1989.

[7] T. Chapman, A. Hayes, M. Tilden, "Reactive maze solving with a biologically inspired wind sensor," in Proc. of the $6^{\text {th }}$ Int.Conf. on Sim. of Adaptive Behavior, 2000, Paris, France, pp.81-87, MIT Press.

[8] M. Fend, S. Bovet, and V. Hafner, "The Artificial Mouse - A Robot with Whiskers and Vision," in Proceedings of the $35^{\text {th }}$ International Symposium on Robotics, Paris, March 2004.

[9] M. Fend, H. Yokoi, and R. Pfeifer, "Optimal morphology of a biologically-inspired whisker array on an obstacle-avoiding robot," in Proc. of the $7^{\text {th }}$ Eur. Conf. on Artificial Life, 2003, pp. 771-780.

[10] D. Jung and A. Zelinski, "Whisker-based mobile robot navigation," in Proc. IEEE/RJS Int. Conf. on Intelligent Robots and Systems, vol. 2, pp. 444-449, 1996.

[11] N. Cowan, E. Ma, M. Cutkosky, and R. Full, "A biologically inspired passive antenna for steering control of a running robot," Int. Symposium on Robotics Research, Siena, Italy, 2003.

[12] M. Kaneko, N. Kanayama, and T. Tsuji, "Active antenna for contact sensing," IEEE Trans. Robotics and Automation, vol.14, pp.278-291, 1998.

[13] G. Scholz and C. Rahn, "Profile sensing with an actuated whisker," IEEE Trans. on Robotics and Automation, vol. 20, pp.124-127, 2004.

[14] R. Russell and J. Wijaya, "Object location and recognition using whisker sensors," Australian Conference on Robotics and Automation, CD-ROM Proceedings ISBN 0-9587583-5-2, 2003.

[15] M. Lungarella, V. Hafner, R. Pfeifer, and H.Yokoi, "An artificial whisker sensor for robotics," in Proc. IEEE Int. Conf. on Intelligent Robots and Systems, 2002, pp. 2931-2936.

[16] M. Lungarella, V. Hafner, R. Pfeifer, H. Yokoi, "Whisking: an unexplored sensory modality," in Proc. $7^{\text {th }}$ Int. Conf. of the Simulation of Adaptive Behavior, 2002, pp. 58-59, MIT Press.

[17] M. Fend, S. Bovet, H. Yokoi, and R. Pfeifer, "An active artificial whisker array for texture discrimination," in Proc. IEEE/RSJ Int. Conf. on Intel. Robots and Systems, Las Vegas, 2003, vol. II, pp. 1044-1049.

[18] V. Hafner, M. Fend, M. Lungarella, R. Pfeifer, P. König, and K. Körding, "Optimal coding for naturally occurring whisker deflections," Proc. $10^{\text {th }}$ Int. Conf. on Neural Information Processing, 2003, pp. 805812.

[19] S. Bovet, M. Fend, and R. Pfeifer(2004), "Simulating whisker sensors - on the role of material properties for morphology, behavior and evolution," in Proc. $8^{\text {th }}$ Int. Conf. on the Simulation of Adaptive Behavior, 2004, Los Angeles, p. 122 - 130.

[20] M. Fend, R. Abt, M. Diefenbacher, S. Bovet, and M. Krafft, "Morphology and Learning - A Case Study on Whiskers," in Proc. $8^{\text {th }}$ Int. Conf. on the Simulation of Adaptive Behavior, 2004, Los Angeles, pp. $114-122$.

[21] M. Thajchayapong and MJ. Hartmann, "Flexural characteristics of rat vibrissae may enable object distance detection," Journal of Neuroscience, in preparation.

[22] S. Goldberg, M. Maimone, L. Matthies, "Stereo vision and rover navigation software for planetary exploration," IEEE Aerospace Proc. Big Sky, MT.

[23] A. Eisenman, C. Liebe, M. Maimone, M. Schwochert, R. Willson, "Mars exploration rover engineering cameras," SPIE Remote Sensing Proc. Toulouse, France, 2001.

[24] E. Baumgartner, H. Aghazarian, A. Trebi-Ollennu, "Rover localization results for the FIDO rover," Proc. SPIE Photonics East, 2001.

[25] Personal communication from Dr. Robert Anderson, planetary geologist and investigation scientist for MER.

[26] http://astrogeology.usgs.gov/Projects/MER-AthenaMI/microscopic_im 